\title{
Percent Recovered
}

National Cancer Institute

\section{Source}

National Cancer Institute. Percent Recovered. NCI Thesaurus. Code C85777.

The percentage of the total dose that is recovered. 